\title{
INTERVENCIÓN PSICOFISIOLÓGICA PARA EL MANEJO AFECTIVO DE UNA PACIENTE CON TRASTORNO DEPRESIVO BIPOLAR-II
}

\author{
David Alberto Rodríguez-Medina y Leticia Ríos Velasco-Moreno \\ UNAM, UACJ \\ México
}

\begin{abstract}
RESUMEN
Se presenta el caso de una paciente femenina de 25 años de edad, soltera, con problemas de pareja y diagnóstico depresivo bipolar tipo II. Para el manejo afectivo de su depresión se utilizaron estrategias de intervención psicofisiológicas que regularan la actividad autonómica subyacente al nivel de estrés, ansiedad y depresión, así como la promoción del lenguaje afectivo mediante la escritura emocional autorreflexiva, y estrategias cognitivo-conductuales como entrenamiento asertivo, solución de problemas y activación conductual. Los resultados de regulación autonómica muestran cambios clínicos en la temperatura periférica en manos para el manejo de estrés; un decremento en la tasa respiratoria cuando la paciente logra expresar su lenguaje afectivo de manera asertiva e incremento de lenguaje propositivo. Los índices psicométricos de ansiedad y depresión disminuyeron clínicamente. Se concluye que el manejo psicofisiológico es útil para regular el estado afectivo negativo y se propone ampliar su manejo posterior con estrategias de aceptación y compromiso.
\end{abstract}

\section{Palabras Clave:}

trastorno bipolar tipo II, temperatura periférica, escritura emocional autorreflexiva, tasa respiratoria, lenguaje afectivo

\section{PSYCHOPHYSIOLOGICAL INTERVENTION FOR THE AFFECTIVE MANAGEMENT OF A PATIENT WITH BIPOLAR DEPRESSIVE DISORDER-II}

\begin{abstract}
We present the case of a 25 -year-old female patient, single, with partner problems and a bipolar type II depressive diagnosis. For the affective management of their depression, psychophysiological intervention strategies were used to regulate the autonomic activity underlying the level of stress, anxiety and depression, as well as the promotion of affective language through self-reflexive emotional writing, and cognitive-behavioral strategies such as assertive training. problem solving and behavioral activation. The results of autonomic regulation show clinical changes in the peripheral temperature in hands for the management of stress; a decrease in the respiratory rate when the patient is able to express her affective language in an assertive manner and increase in proactive language. The psychometric scores of anxiety and depression decreased clinically. It is concluded that the psychophysiological management is useful to regulate the negative affective state and it is proposed to extend its subsequent management with acceptance and commitment strategies.
\end{abstract}

Keywords:

type II bipolar disorder, peripheral temperature, self-reflective emotional writing, respiratory rate, affective language

Bitácora del Artículo:

| Recibido: 1 de Febrero de 2019 | Aceptado: 1 Abril de 2019 | Publicado en línea: Enero-Junio de 2019 | 


\title{
INTERVENCIÓN PSICOFISIOLÓGICA PARA EL MANEJO AFECTIVO DE UNA PACIENTE CON TRASTORNO DEPRESIVO BIPOLAR-II
}

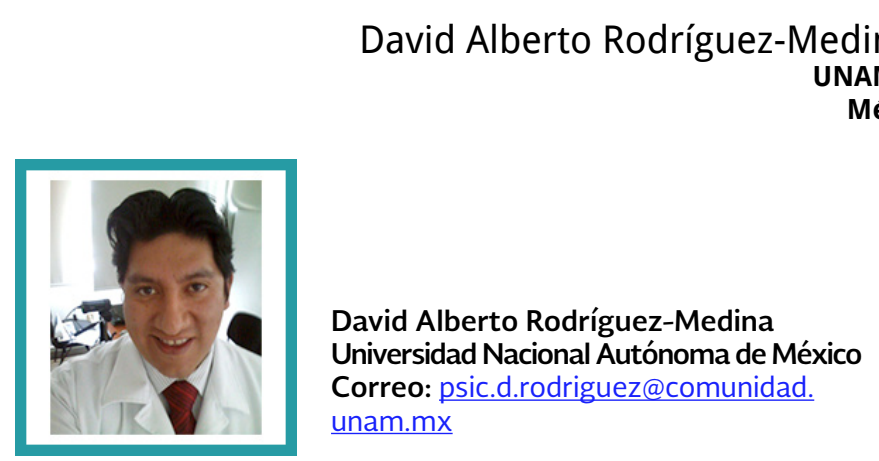

Doctor en Psicología y Salud por la UNAM. Autor de más de 20 artículos en revistas indexadas nacionales e internacionales, con más de 30 conferencias. Revisor metodológico de tesis de licenciatura, así como revisor de artículos en revistas de investigación psicológica especializada

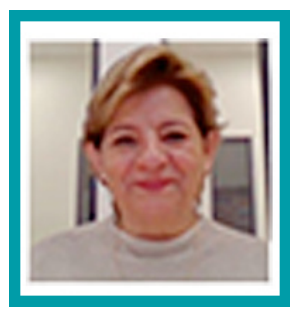

Leticia Ríos Velasco-Moreno Universidad Autónoma de Ciudad Juárez Correo: Irios@uacj.mx

Doctora en Psicología por la Universidad de Málaga. Profesora investigadora titular C de tiempo completo, UACJ

\section{CONTRIBUCIÓN DE LOS AUTORES}

David Alberto estuvo a cargo de la evaluación e intervención psicofisiológica, análisis de resultados y redacción de estudio.| Leticia Ríos participó como coterapeuta.

\begin{abstract}
AGRADECIMIENTOS
Por su invaluable apoyo moral, afectivo e instrumental: a la Psic. Gabriela Jael Pérez García, la Mtra. Gloria García, el Lic. Roberto Pérez Sánchez, la Lic. Elizabeth Hernández García, el Psic. Daniel Pérez García. Un agradecimiento especial a Irma Rodríguez Sosa, Lucianita Sosa, Frida Rodríguez, Dr. Leonardo Rodríguez Sosa, Dr. Leonardo Rodríguez Medina y la Lic. Ma. Estela Medina Hernández. Agradecemos también al Dr. Benjamín Domínguez y el grupo Mente-Cuerpo de la Facultad de Psicología: Andrea Herrera, Gerardo Leija, Esael Pineda, Erik Mateos, Sandra Vergara, Mariana Ortíz, Omar Chavarría, Sara Pluma, Viridiana Peláez y Li Erandi. Al grupo Psicología Social de la Salud de la uam-i : Omar Manjarrez, Mario Carranza y Joél Vázquez. A los ingenieros en mecatrónica de la uaq, el Dr. Luis Alberto Morales Hernández y el Dr. Irving Cruz Albarrán. Por último, a la Dra. Rebeca Robles García, del inprfm y a la Dra. Rocío Hernández Pozo, del crim-unam.
\end{abstract}

\section{DATOS DE Filiación de los Autores}

Universidad Nacional Autónoma de México | Universidad Autónoma de Ciudad Juárez

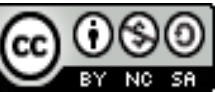

Copyright: (c) 2019 Rodríguez-Medina, D. A. \& Velasco-Moreno, L.R.

Este es un artículo de acceso abierto distribuido bajo los términos de la licencia Creative Commons Reconocimiento-NoComercial 4.0 Internacional, por lo que su contenido gráfico y escrito se puede compartir, copiar y redistribuir total o parcialmente sin necesidad de permiso expreso de sus autoras con la única condición de que no se puede usar con fines directamente comerciales y los términos legales de cualquier trabajo derivado deben ser los mismos que se expresan en la presente declaración. La única condición es que se cite la fuente con referencia a la Revista Digital Internacional de Psicología y Ciencia Social y a sus autoras. 


\section{TABLA DE CONTENIDO}

INTRODUCCIÓN

MÉTODO

Diseño., 6

Participantes., 6

Instrumento., 7

Procedimiento., 7

Resultados, 7

CONCLUSIONES

Discusión

REFERENCIAS 


\section{INTRODUCCIÓN}

$\mathrm{F}$ I trastorno depresivo bipolar (TDBP) se define como episodios oscilantes del estado anímico extremo en manía (o hipomanía en BP-II) y depresión (American Psychiatric Association, 2013). Por lo común se presenta durante la adolescencia o la adultez temprana antes de los 25 años. Si se presenta en una edad superior de los 50 años se debe sospechar de un problema orgánico (Suárez, Montes, Jarabo y Forteza, 2015).

La prevalencia mundial del TDBP es de casi 1\% (García-Blanco, Sierra y Livianos, 2014). Se estima que en México afecta entre 1 y 3\% de la población, y en 2017 alcanzó 15\% de las consultas externas del Hospital Psiquiátrico Fray Bernardino Álvarez (Secretaría de Salud, 2018).

Las manifestaciones clínicas del TDBP incluyen hipomanía, depresión, ansiedad, estrés, presión arterial alta (Alfaro, Álvarez, Castaño, Medina, Montoya, Ortega y Valencia, 2015). La manía se manifiesta como exaltación, disminución del sueño, autoestima muy elevada, sobrexcitación, hostilidad, bajo control de temperamento e irritabilidad, mientras los síntomas depresivos incluyen baja autoestima, abatimiento, desesperanza, minusvalía, sentimientos de culpa, y problemas de sueño, concentración y alimentación (Secretaría de Salud, 2018). Se ha relacionado comorbilidad con trastornos por abuso de sustancias, trastornos de ansiedad, trastornos por y de la personalidad. El DSM-5 contempla la posibilidad de especificar si presenta ansiedad para la planeación del tratamiento (García-Blanco et al., 2014).

Los tratamientos clínicos disponibles que han mostrado eficacia para la reducción de la sintomatología depresiva del TDBP son farmacológicos, en especial los estudios clínicos aleatorizados del efecto de la ketamina (Alberich, Martínez-Cengotitabengoa, López, Zorrilla, Núñez, Vieta y González-Pinto, 2017; McGirr, Berlim, Bond, Fleck, Yatham y Lam, 2014), así como las intervenciones psicológicas, en particular la psicoeducación, la terapia cognitivo/conductual, terapia cognitiva basada en la atención plena, terapia dialéctica conductual (Cakmak, Sut, Ozturk, Tamam y Bal, 2016) y la Terapia Ocupacional (Rodríguez y Montero, 2017). Si bien hay diversas estrategias psicológicas para el tratamiento del paciente con TDBP (Ngazimbi, Lambie y Shillingford, 2008), no se han incorporado medidas psicofisiológicas en la mayoría de estas intervenciones, cuyo su efecto psicofisiológico existe si la sintomatología de estrés, an- siedad y depresión disminuye en el paciente. Escasos de estudios han considerado los tratamientos psicofisiológicos (Katsamanis, Lehrer, Escobar, Gara, Kotay y Liu, 2011) para el control de la sintomatología del paciente TDBP (Henry, Minassian, Paulus, Geyer y Perry, 2010; Vega y González, 2017), encontrando efectos positivos cardiovasculares en el manejo de estrés.

Entre los objetivos de las estrategias psicológicas se encuentra la reducción de sintomatología de estrés y ansiedad, tradicionalmente evaluados mediante medidas psicométricas como el Inventario de Ansiedad de Beck (Stratford, Cooper, Di Simplicio, Blackwell y Holmes, 2015). Sin embargo, se desconoce si la intervención psicofisiológica de regulación autonómica térmica como las estrategias de relajación y la expresión emocional disminuyen la sintomatología de distrés psicológico del TDBP. Estudios recientes (Rodríguez-Medina y Domínguez, 2017; Rodríguez, Domínguez, Cortés, Cruz, Morales y Leija, 2018) presentaron evidencia del efecto de la relajación sobre periférica en manos y nariz en población clínica y no clínica. En la figura 1 se muestra el efecto de la respiración diafragmática en una persona saludable.
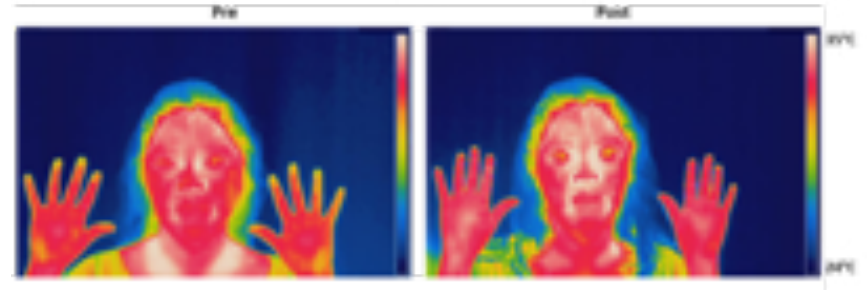

Figura 1.

Efecto de la respiración diafragmática en la temperatura periférica en manos y nariz en una persona saludable (Rodríguez-Medina, Domínguez, Cortés, Cruz, Morales y Leija, 2018). La expresión emocional y el entrenamiento en respiración diafragmática incrementa la temperatura periférica facial y en manos.

A continuación se presenta el estudio caso del tratamiento psicológico de una paciente con TDBP cuyo propósito fue la intervención psicofisiológica para la disminución de sintomatología de ansiedad (puntajes psicométricos) y estrés (medición de temperatura periférica en manos) mediante estrategias cognitivo-conductuales, relajación y expresión emocional.

\section{Método}

\section{Identificación del caso}

La paciente CR es una femenina de 25 años, chef, soltera con problemas en su relación de pareja desde hace dos 
años, con Dx Trastorno Depresivo Bipolar-II, en fase de depresión, con comorbilidad sintomática de episodios de ansiedad. Aún cuando su estado afectivo fue predominantemente depresivo, al hablar de sus aspiraciones profesionales mostró una actividad muy competitiva para buscar una beca de estudio en el extranjero (hipomanía). Por medio de la entrevista clínica se exploró la sintomatología de los trastornos afectivos. Durante la fase de evaluación se consideraron los criterios diagnósticos del CIE-10: F31.8 Trastorno bipolar II (296.89).

- A. Historia de algunos episodios depresivos mayores.

- B. Presencia e historia de episodios hipomaníacos (sin alteraciones funcionales).

- C. Sin presencia de manía (crisis psicóticas o disfunciones sociales).

- D. Ningún otro trastorno afectivo explica mejor su comportamiento.

- E. Los síntomas le producen malestar significativo para resolver problemas de su interés.

No ingirió medicamentos durante el tratamiento. Se presentó con alineo y orientación mental.

\section{Análisis del motivo de la intervención}

CR acude a consulta porque ha experimentado sentimientos de inestabilidad emocional personal, desesperanza con la pareja. La paciente reporta ser hostil, con problemas de comunicación afectiva cuando tiene problemas con su pareja, depresión, aislamiento social y desequilibrio del ciclo menstrual. En el pasado reciente buscó relaciones pasajeras que le proveyeran algún nivel de satisfacción, pero decidió optar por retomar su relación de pareja. Sostiene ideas de grandeza creando retos personales concentrados en planes futuros, como expandir sus conocimientos para viajar a estudiar; muestra deficiencias en la toma de perspectiva orientada al otro y reacción emocional. Refiere estar en una actitud defensiva, y cuando no obtiene lo esperado en la pareja muestra una actitud pasivo-agresivo para ver si su pareja le da la respuesta que espera. Sin embargo, la relación parece tan desgastada que ya no encuentra esa respuesta.

\section{Antecedentes del problema}

Recientemente falleció su abuela materna, una figura importante en su vida porque a la falta de atención de la madre desde los cinco años por problemas económicos y personales. Lleva una vida en pareja disfuncional, pues la pareja muestra desesperanza aprendida de continuar con la relación. Dos años antes de la consulta psicológica tuvo un aborto en el segundo mes de embarazo, por lo que la paciente presenta sentimientos de soledad, culpa y necesidad de apoyo de su pareja. Al no recibirlo buscó relaciones interpersonales pasajeras para obtener el afecto que disminuya su sintomatología ansiosa y depresiva.

\section{Descripción y análisis del problema}

Sus principales estresores han sido las idealizaciones de pareja y la incapacidad de expresar emociones y sentimientos con el que ha sido su pareja y familia, así como deficiencias en la resolución de problemas, empatía y conductas impulsivas (audios 1 y 2). En la figura 2 se muestra el mapa psicopatogénico de la paciente.

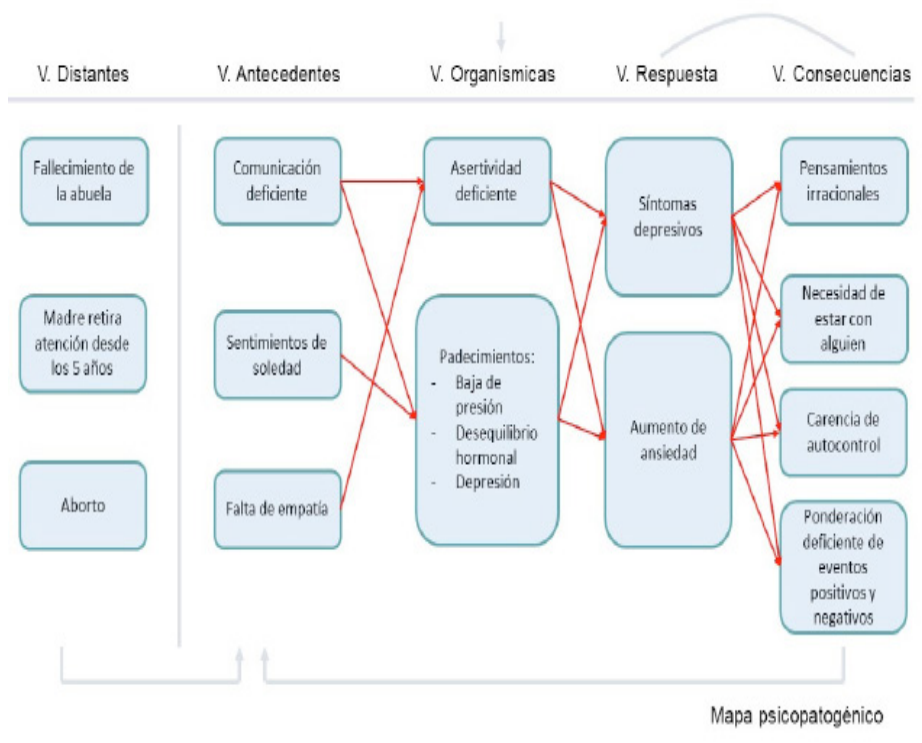

Figura 2.

Mapa psicopatogénico de la paciente. Las variables organísmicas influyen directamente en las variables respuesta, y éstas en sus consecuencias, lo cual retroalimenta los antecedentes de las manifestaciones clínicas, cerrando así el circuito. A partir de las variables organísmicas se decide abrir en el tratamiento psicofisiológico propuesto.

\section{Plan de intervención}

\section{Descripción del plan intervención}

Se procedió a implementar un plan de tratamiento basado en la expresión emocional y control de las cogniciones irracionales mediante reestructuración cognoscitiva y activación conductual. El plan de tratamiento (Tx) tuvo una duración de ocho meses, una sesión semanal con una duración de una hora en consultorio privado. Las técnicas que se utilizaron fueron: reestructuración cognitiva, solución de problemas, autocontrol, activación conductual y entrenamiento asertivo (figura 3). 


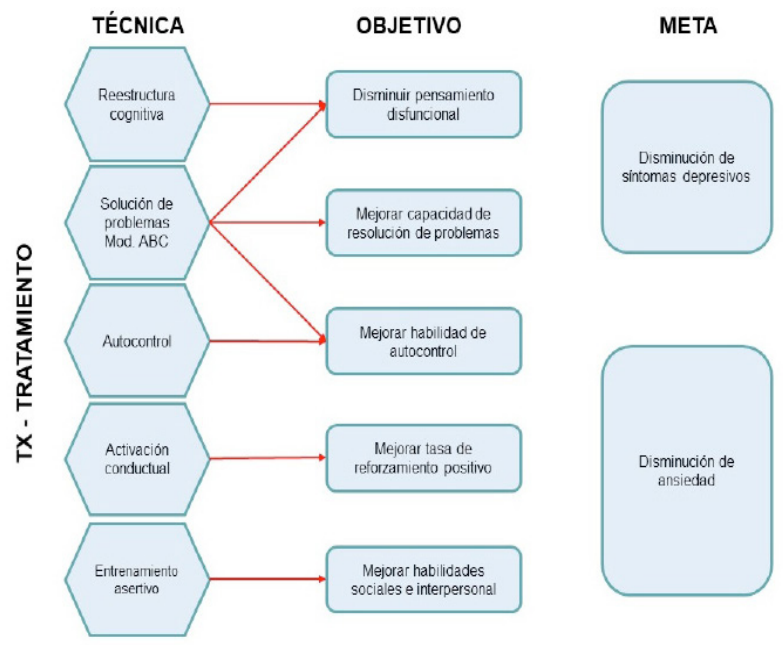

Figura 3.

Plan de tratamiento psicológico (mapa de alcance de metas). EI tratamiento psicofisiológico propuesto se enfoca en las variables organísmicas para su regulación autonómica y de expresión emocional que revierta la sintomatología depresiva y ansiosa.

\section{Establecimiento de los objetivos y las metas} del plan de intervención

Disminuir los síntomas de depresión (pensamiento disfuncional, mejorar capacidad de resolución de problemas, mejorar y promover la expresión emocional, mejorar índice de reforzamiento positivo) y de ansiedad (regulación autonómica afectiva mediante el incremento de temperatura periférica y decremento de la tasa respiratoria con entrenamiento en relajación y habilidades sociales).

Inventario de Ansiedad de Beck (BAI). Instrumento de autorreporte con 21 ítems donde se califica la intensidad de los síntomas de ansiedad. Las opciones de respuesta van de 0 a 3 puntos. Cuenta con buenas propiedades psicométricas, validado para población mexicana (Robles, Varela, Jurado y Paez, 2001).

Inventario de Depresión de Beck (BDI). Instrumento de tamizaje autoaplicable elaborado con 21 reactivos con respuestas tipo Likert que van de 0 a 3 puntos. Se consideraron los puntos de corte de acuerdo con Jurado, Villegas, Méndez, Rodríguez, Loperena y Varela (1998). Aun cuando existen informes más recientes acerca de sus propiedades psicométricas, se concuerda que el punto de corte mínimo de sintomatología depresiva es de nueve (9).

\section{Técnicas de evaluación del plan de intervención}

Para evaluar la sintomatología de ansiedad y depresión se contó con los puntajes pre-post de Inventario de Ansiedad de Beck (BAI) e Inventario de Depresión de Beck (BDI-II), respectivamente, cuyas propiedades psicométricas han sido validadas en población mexicana.
Para la valoración de estrés se utilizó el registro de la tasa respiratoria y la temperatura periférica mediante el equipo desarrollado por Domínguez y Rodríguez-Rossini (2002, citado en Memorias del Premio Universitario León Bialik a la Innovación Tecnológica, 2011) en manos pre-post $\mathrm{Tx}$, utilizando el perfil psicofisiológico de estrés (Domínguez, Olvera, Cruz y Cortés, 2001).

Para promover la expresión emocional se utilizó la Escritura Emocional Autorreflexiva (EEA), la cual permite evaluar el lenguaje emocional según su valencia positiva y negativa. Dicha técnica promueve un lenguaje afectivo que induce cambios en la reestructuración cognitiva (Olvera, Domínguez, Cruz, Pennebaker y Cortés, 2002; Dzib-Aguilar, Moo-Estrella y Chan-Coob, 2010). Esta técnica consta de cuatro ejercicios escritos: 1) se solicita al paciente que escriba alguna experiencia que le haya causado malestar y que no le hayan contado a alguien (ejercicio A); 2) se le indica que vuelva escribir la misma experiencia, pero que incorpore la mayor cantidad de palabras que le acomoden a su texto de una lista de palabras afectivas que se le proporciona y se dejan al alcance de su vista (ejercicio B); 3) se le pide que escriba la misma experiencia, pero ahora narrada en tercera persona (ejercicio C), y 4) se solicita al paciente que escriba lo que aprendió de esa experiencia (ejercicio D). En cada ejercicio se monitoreó la temperatura periférica durante la aplicación de la técnica EEA pre-post Tx.

\section{EFICACIA dEL PLAN DE INTERVENCIÓN}

\section{Aplicación del plan de intervención}

De las técnicas descritas se trabajó con mayor énfasis la reestructuración cognoscitiva, al ser la parte cognitiva de la terapia la que - según la literatura — mayor eficacia ha demostrado en pacientes deprimidos. Sin embargo, en el resto de las técnicas se trataron y lograron los objetivos planteados, con excepción de la profundización en autocontrol

\section{Presentación de resultados}

Primero se obtuvo una valoración psicométrica pre-post Tx de la paciente CR. Los resultados se muestran en la tabla 1. Después se presenta el perfil psicofisiológico de estrés pre-post (figura 4). Durante las primeras cuatro sesiones la paciente reportó registros ACC continuamente. En éstos su lenguaje emocional predominante fue negativo, con mayor número de quejas que demandas, sobre todo externas. En seguida redujo los registros traídos a la sesión y con ello su lenguaje emocional negativo. Concedió menor valor a los eventos estresantes cotidianos a partir de la quinta sesión y hasta el término del Tx. 

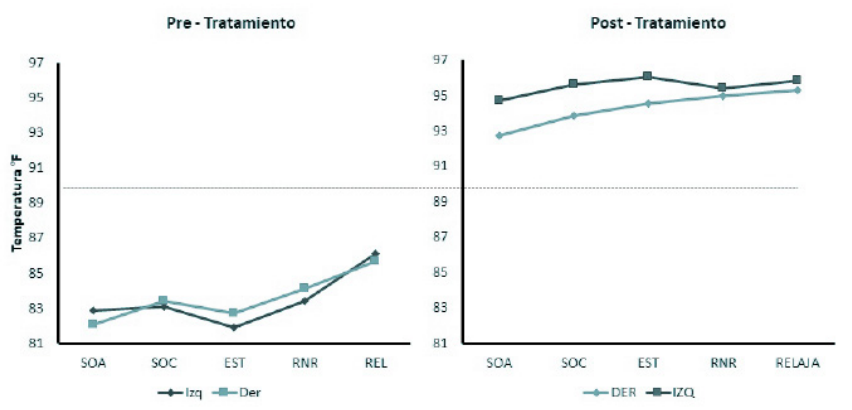

Figura 4.

Perfil psicofisiológico de estrés pre-post Tx. Temperatura periférica bilateral. SOA = sentado con ojos abiertos; $S O C=$ sentado con ojos cerrados, EST = estresor afectivo (pensamiento negativo); $R N R=$ respuesta natural de relajación; $R E L A J A=$ inducción de la relajación mediante respiración diafragmática. La línea punteada indica el valor de referencia para considerar predominancia autonómica simpática/parasimpática: entre 79-85 ${ }^{\circ} \mathrm{F}$ se considera predominio de actividad simpática; entre $86-90^{\circ} \mathrm{F}$ se considera alta actividad simpática y baja parasimpática; por encima de $90^{\circ} \mathrm{F}$ se considera alta actividad parasimpática con baja actividad simpática, y por arriba de $94{ }^{\circ} \mathrm{F}$ se considera predominio de actividad parasimpática.

Tabla 1.

Puntajes psicométricos.

\begin{tabular}{|c|c|c|}
\hline & Pre & Post \\
\hline Ansiedad (BAI) & 44 (Muy alta) & 16 (Muy baja) \\
\hline Depresión (BDI) & 26 (Moderada) & 8 (Sin depresión) \\
\hline
\end{tabular}

\section{Análisis de los cambios que produjo el plan de intervención}

La temperatura periférica bilateral es un indicador de la actividad simpática y parasimpática que observar el incremento de la condición basal a la actual para manejar sus estresores. Como criterios de cambio clínico se utilizaron los propuestos por Domínguez et al. (2001). Como se muestra en el primer perfil psicofisiológico (figura 4), la px presenta dominancia en tres de las cinco fases (SOC, EST y RNR). Muestra ganancia después de EST y sincronía después de SOC. La intervención psicológica en la fase REL fue mediante un ejercicio computarizado de respiración diafragmática que incrementó de manera notable sus temperaturas, así como la simetría y sincronía. Para el segundo perfil psicofisiológico de estrés aumenta de modo significativo sus temperaturas, perdiendo dominancia, pero contando con ganancia; la sincronía se mantiene hasta RNR, donde aún se apre- cian dificultades para relajarse por sí misma, pero que retoma con la intervención psicológica mediante el mismo ejercicio de respiración diafragmática (RELAJA).

\section{Significancia clínica de los cambios}

Clínicamente la px ya no concede la misma importancia a los problemas que tiene de manera cotidiana y los comienza a expresar en su magnitud adecuada. Para ello se trabajó con la técnica de escritura emocional autorreflexiva (EEA) - a continuación se muestran los cambios psicofisiológicos y tasa de palabras emocionales positivas y negativas asociados a los cambios autonómicos en cada ejercicio pre-post Tx psicológico (figura 5)—. Con base en los criterios de Domínguez et al. (2001), CR presenta dificultad para expresar sus emociones negativas cuando disminuye su temperatura periférica bilateral, encontrando asimetría, asincronía y pérdida de temperatura en el ejercicio B antes del tratamiento. Sin embargo, después del tratamiento cumple los criterios de significancia clínica: ganancia, es decir, aumenta su temperatura bilateral por encima de los $94{ }^{\circ} \mathrm{F}$, mantiene sincronía y asimetría (las temperaturas se comportan de manera semejante entre sí y la distancia entre ellas es menor a $1{ }^{\circ} \mathrm{F}$ ).
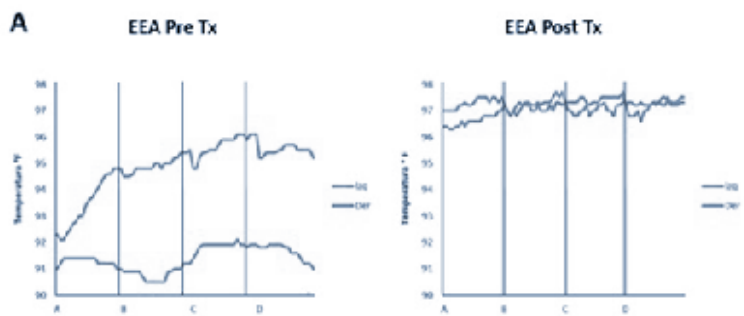

B
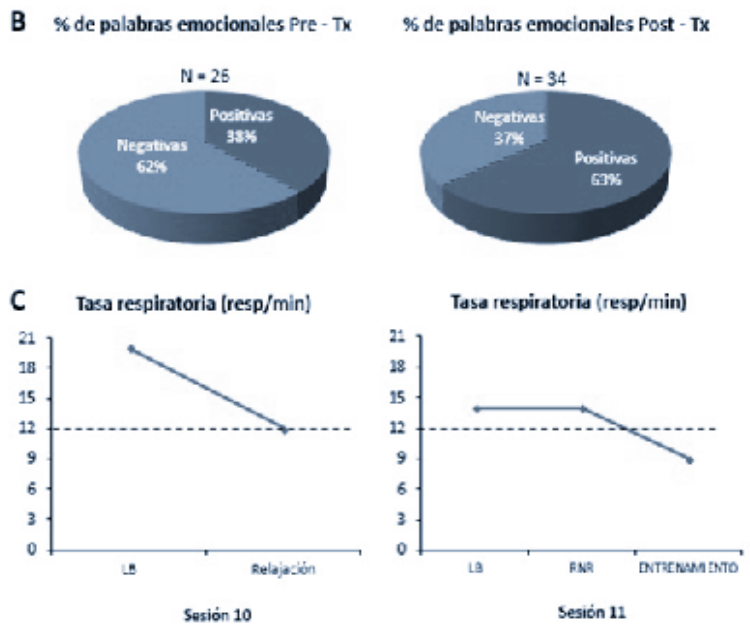

Figura 5.

Efectos psicofisiológicos de la escritura emocional autorreflexiva en la temperatura periférica y el lenguaje afectivo pre-post $T x$. (A) Efectos de la escritura emocional autorreflexiva (EEA) pre-post tratamiento. (B) Lenguaje afectivo de la EEA pre-post 
tratamiento. (C) Tasa respiratoria en relajación mediante respiración diafragmática y entrenamiento asertivo (la línea punteada indica el valor de referencia clínico para establecer una respiración adecuada). $L B=$ línea base, $R N R=$ respuesta natural de relajación, Entrenamiento $=$ entrenamiento asertivo

Los cambios autonómicos muestran una estrecha relación con el lenguaje emocional de CR. Aun cuando su lenguaje emocional fuera negativo, el valor de sus estresores disminuyó. En las evaluaciones intermedias del Tx psicológico mostró este patrón de respuestas, y se tomó su tasa respiratoria para desarrollar el lenguaje interoceptivo cuando está estresada y cuando está relajada, aun cuando su repertorio verbal fuera negativo. Se tomó su LB respiratoria después de comunicar sus emociones negativas y se intervino con una técnica de relajación muscular progresiva. El punto de corte para el control respiratorio máximo (respiración diafragmática) es de 12 respiraciones por minuto, mismo que se alcanzó después del entrenamiento en relajación.

\section{Relevancia social del cambio}

Las estrategias de regulación autonómica permiten regular los estados afectivos negativos de largo plazo. En el caso que CR enfrenta, que presenta TDBP, alcanzará a modular sus experiencias negativas que enfrente en sus proyectos de vida. Es posible que logre sus objetivos personales más importantes según su significación. Sin embargo, es necesario un monitoreo de su estado afectivo, su manejo conductual y las consecuencias afectivas de éste. También es necesario estudiar si logra mantener una relación de pareja estable, el entrenamiento en habilidades sociales que promuevan la empatía, pues sustituir los compromisos rotos por ideas de grandeza no favorecerá la relación interpersonal de largo plazo, aspecto significativo para la vida de la paciente. Se podría incluso recomendar estrategias de aceptación y compromiso que alienten las prioridades que ella sostenga en cada etapa de su vida, continuando con las estrategias de regulación autonómica, como la expresión emocional asertiva y las estrategias de relajación.

\section{Pronóstico}

\section{Descripción de la fase de seguimiento}

Los resultados indican que para CR es necesaria la expresión emocional de los estresores que la aquejan; el desarrollo de lenguaje interoceptivo que le permita reconocer cuando algo la perturba y desarrollar las habilidades necesarias para la relajación por sí misma, aunado a los cambios cognitivos que se desarrollaron durante la terapia, los recursos socioemocionales y conductuales que le benefician en esparcir su repertorio de actividades a desarrollar y no enfocar su fuente de reforzamiento en una idealización. Las palabras emotivas se incrementaron y con ello la relación de palabras con valencia negativa se invirtió en proporción 3:1 positivas luego del tratamiento. Se requiere trabajar con la modificación de algunos pensamientos disfuncionales una vez expresadas las emociones para su manejo ulterior. Es necesario trabajar en entrenamiento de solución de problemas contando con todos los recursos al alcance de la paciente: afectivos (expresión emocional), conductuales (nuevas actividades) y cognitivos (revalorar las percepciones iniciales de eventos emocionales).

Aunado a esto se recomienda continuar con los ejercicios propuestos durante el Tx para desarrollar las habilidades de autocontrol que le generen mayores ganancias a largo plazo respecto a su salud y conductas no empáticas emocionalmente para un fin común. Así como también reorganizar los valores de sus actividades para considerar sus prioridades continuamente con el monitoreo emocional. Como sugerencia terapéutica, para el manejo de la empatía es necesario ponderar qué siente el otro que puedo hacer yo para empatar y desarrollar el objetivo que demandan mis sentimientos. Cuando no se tenga certeza de cómo actuar la conducta imitativa (como se indicó en el FEEL Test), puede favorecer los lazos de contagio emocional y desarrollar el componente empático afectivo.

La evaluación psicofisiológica de temperatura bilateral del perfil psicofisiológico de estrés, así como los del ejercicio de EEA pre-post intervención que se comentan en los resultados, fueron tomados durante las primeras tres sesiones del tratamiento y tres sesiones previas al cierre del mismo (figuras 5 A y B). Sólo se registró la tasa respiratoria durante el tratamiento (figura $5 \mathrm{C}$ ) para valorar los cambios clínicos en la respiración durante el entrenamiento asertivo de la expresión emocional y la relajación, mostrando que las estrategias mencionadas redujeron clínicamente la respuesta somática.

\section{Resultados en la fase de seguimiento}

La paciente cuenta con herramientas que le proporcionan un estilo de afrontamiento adaptativo y reactividad emocional, medida por la actividad simpática de la temperatura periférica bilateral; su lenguaje positivo ante los eventos estresantes disminuyó a lo largo del Tx psicológico. Se sugiere dar seguimiento durante un periodo de largo plazo que permita determinar si los efectos del entrenamiento en regulación afectiva autonómica (rela- 
jación y expresión) se conservan o requerirá de apoyo psicológico para el control de esta sintomatología.

\section{Referencias}

Alberich, S., Martínez-Cengotitabengoa, M., López, P., Zorrilla, I., Núñez, N., Vieta, E., \& González-Pinto, A. (2017). Eficacia y seguridad de la ketamina en depresión bipolar. Una revisión sistemática. Revista de Psiquiatría y Salud Mental, 10(2), 104-112. http://dx.doi.org/10.1016/j. rpsm.2016.05.005

Alfaro, L., Álvarez, C., Castaño, J., Medina, M., Montoya, M., Ortega, N., \& Valencia, G. (2015). Relación entre trastorno afectivo bipolar, depresión, ansiedad, estrés y valores de presión arterial en población de la Clínica San Juan de Dios, Manizales, Colombia, 2014. Archivos de Medicina (Col), 15(2), 281-290. Recuperado el 4 de julio de 2018 de http://www.redalyc.org/pdf/2738/273843539012.pdf

American Psychiatric Association (2013). Diagnostic and Statistical Manual of Mental Disorders. 5a. ed. Arlington: American Psychiatric Publishing.

Cakmak, S., Sut, H., Ozturk, S., Tamam, L., \& Bal, U. (2016). The Effects of Occupational Therapy and Psychosocial Interventions on Interpersonal Functioning and Personal and Social Performance Levels of Corresponding Patients. Arch Neuropsychiatry, 53(3), 234-240. http://dx.doi. org/10.5152/npa.2015.10130

Domínguez, B., Olvera, Y., Cruz, A., \& Cortés, J. (2001). Monitoreo no invasivo de la temperatura periférica en la evaluación y tratamiento psicológico. Psicología y Salud, 11(2), 6171. Recuperado el 9 de julio de 2018 de https://www. researchgate.net/publication/275951248_Monitoreo_ no invasivo de la temperatura periferica en la evaluacion y tratamiento psicologico

Dzib-Aguilar, J., Moo-Estrella, J., \& Chan-Coob, J. (2010). Técnicas psicoterapéuticas para proteger la confidencialidad y la responsabilidad legal. Implicaciones del Biofeedback y Eeasp en el contexto jurídico. Diversitas. Perspectivas en Psicología, 6(2), 441-448. Recuperado el 9 de julio de 2018 de http://www.redalyc.org/articulo.oa?id=67915140016

García-Blanco, A., Sierra, P., \& Livianos, L. (2014). Nosología, epidemiología y etiopatogenia del trastorno bipolar. Últimas aproximaciones. Psiquiatría Biológica, 21(3), 8994. http://dx.doi.org/10.1016/j.psiq.2014.07.004

Henry, B., Minassian, A., Paulus, M., Geyer, M., \& Perry, W. (2010). Heart rate Variability in Bipolar Mania and Schizophrenia. Journal of Psychiatric Research, 44(3), 168-176. http:// dx.doi.org/10.1016/j.jpsychires.2009.07.011

Jurado, S., Villegas, M. E., Méndez, L., Rodríguez, F., Loperena, V., \& Varela, R. (1998). La estandarización del inventario de depresión de Beck para los residentes de la Ciudad de México. Salud Mental, 21(3), 26-31.

Katsamanis, M., Lehrer, P., Escobar, J., Gara, M., Kotay, A., \& Liu, R. (2011). Psychophysiologic Treatment for Patients with Medically Unexplained Symptoms. A Randomized Controlled Trial. Psychosomatics, 52(3), 218-229. http:// dx.doi.org/10.1016/j.psym.2011.01.015

McGirr, A., Berlim, M., Bond, D., Fleck, M., Yatham, L., \& Lam,
R. (2014). A Systematic Review and Meta-Analysis of Randomized, Double-Blind, Placebo-Controlled Trials of Ketamine in the Rapid Treatment of Major Depressive Episodes. Psychological Medicine, 45(04), 693-704. http:// dx.doi.org/10.1017/s0033291714001603

Memorias del Premio Universitario León Bialik a la Innovación Tecnológica (2011). Recuperado el 9 de julio de 2018 de http://reunicep.com/img/logos/leon-bialik-003.pdf

Ngazimbi, E., Lambie, G., \& Shillingford, M. (2008). The Use of Narrative Therapy with Clients Diagnosed with Bipolar Disorder. Journal of Creativity in Mental Health, 3(2), 157174. http://dx.doi.org/10.1080/15401380802226661

Olvera, Y., Domínguez, B., Cruz, A., Pennebaker, J. \& Cortés, J. (2002). Evaluación de la escritura emocional autorreflexiva en estudiantes de ingeniería del Instituto Politécnico Nacional. Enseñanza e Investigación en Psicología, 7(1).

Robles, R, Varela, R, Jurado, S, \& Paez, F. (2001). Versión mexicana del Inventario de Ansiedad de Beck. Propiedades psicométricas. Revista Mexicana Psicología, 18.

Rodríguez Martínez, M., \& Montero Sánchez, R. (2017). La terapia ocupacional en personas con trastorno bipolar. Revista Chilena de Terapia Ocupacional, 17(1), 91. http:// dx.doi.org/10.5354/0719-5346.2017.46381

Rodríguez-Medina, D., \& Domínguez, B. (2017). La evaluación psicofisiológica con imagen térmica infrarroja en los procesos psicológicos. Revista Digital Internacional de Psicología y Ciencia Social, 3(2), 228-242. http://dx.doi. org/10.22402/j.rdipycs.unam.3.2.2017.140.227-241

Rodríguez Medina, D., Domínguez Trejo, B., Cortés Esteban, P., Cruz Albarrán, I., Morales Hernández, L., \& Leija Alva, G. (2018). Biopsychosocial Assessment of Pain with Thermal Imaging of Emotional Facial Expression in Breast Cancer Survivors. Medicines, 5(2), 30. https://doi.org/10.3390/ medicines5020030

Secretaría de Salud (2018). 048. Trastorno afectivo bipolar, una de las primeras causas de consulta. Recuperado el 4 de julio de 2018 de https://www.gob.mx/salud/prensa/048trastorno-afectivo-bipolar-una-de-las-primeras-causasde-consulta

Suárez, M., Montes, M., Jarabo, D., \& Forteza, G. (2015). Trastorno bipolar. Medicine-Programa de Formación Médica Continuada Acreditado, 11(85), 5075-5085. http:// dx.doi.org/10.1016/j.med.2015.08.003

Stratford, H., Cooper, M., Di Simplicio, M., Blackwell, S., \& Holmes, E. (2015). Psychological Therapy for Anxiety in Bipolar Spectrum Disorders. A Systematic Review. Clinical Psychology Review, 35, 19-34. http://dx.doi.org/10.1016/j. cpr.2014.11.002

Vega, G., \& González, A. (2017). Eficacia de un programa de biorretroalimentación para regular respuestas fisiológicas ante estrés en personas con diagnóstico psiquiátrico. Revista Latinoamericana de Medicina Conductual. 7(2). Recuperado de http://www.revistas.unam.mx/index.php/ rlmc/article/view/62346/54861 


\section{Meta-Análisis del Artículo}




\section{Dimensión Cuantitativa}

\section{Perfil de Evaluación entre pares}
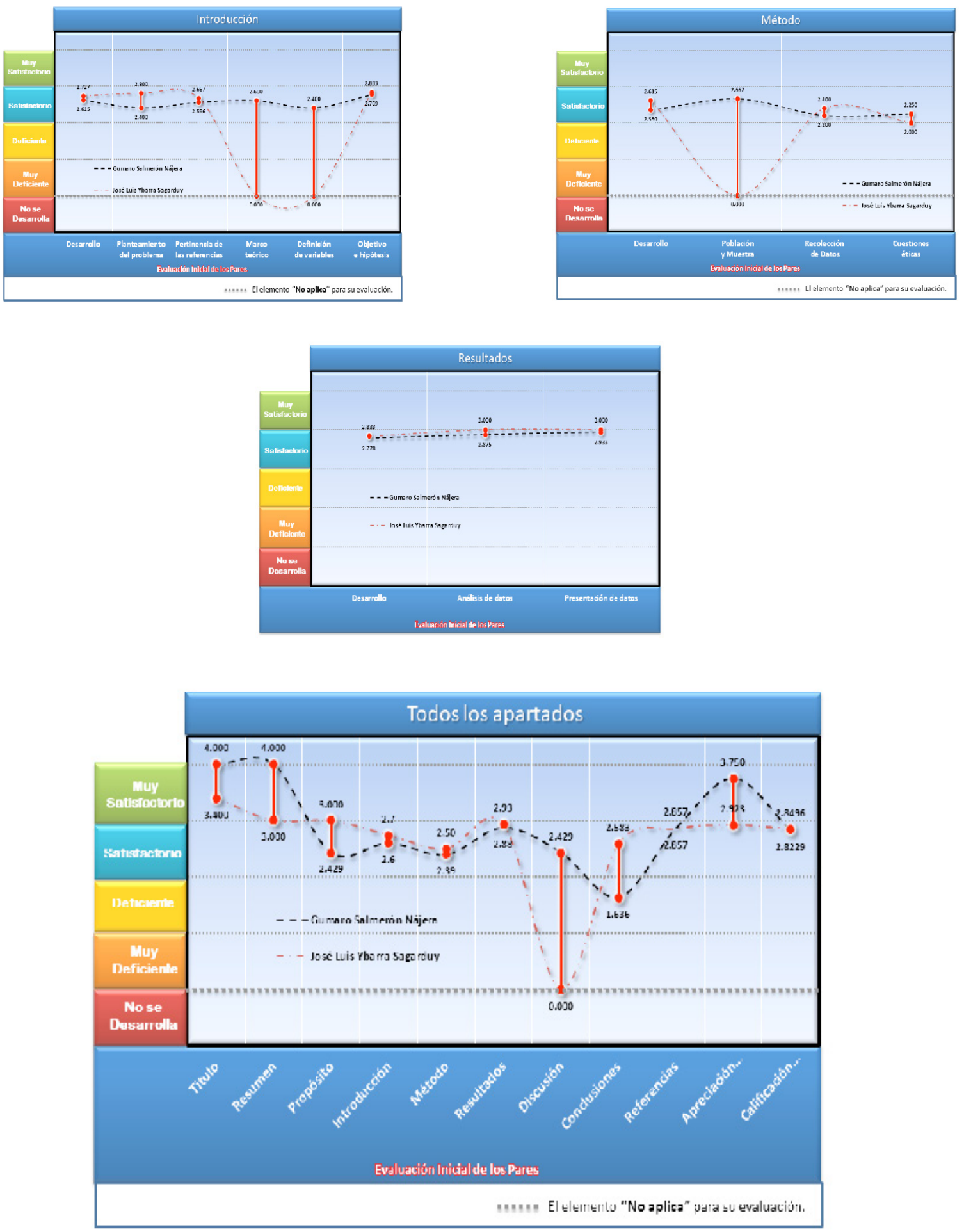

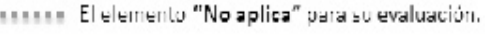




\section{Índice de Concordancia}

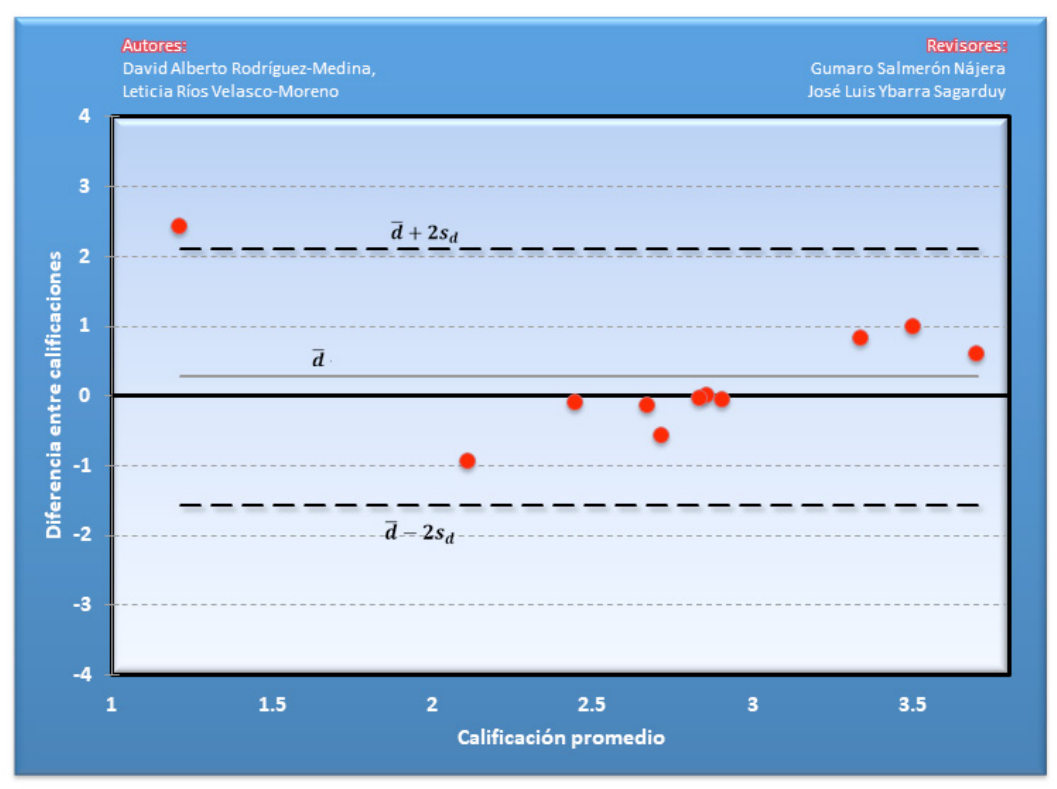

Índice de Acuerdo

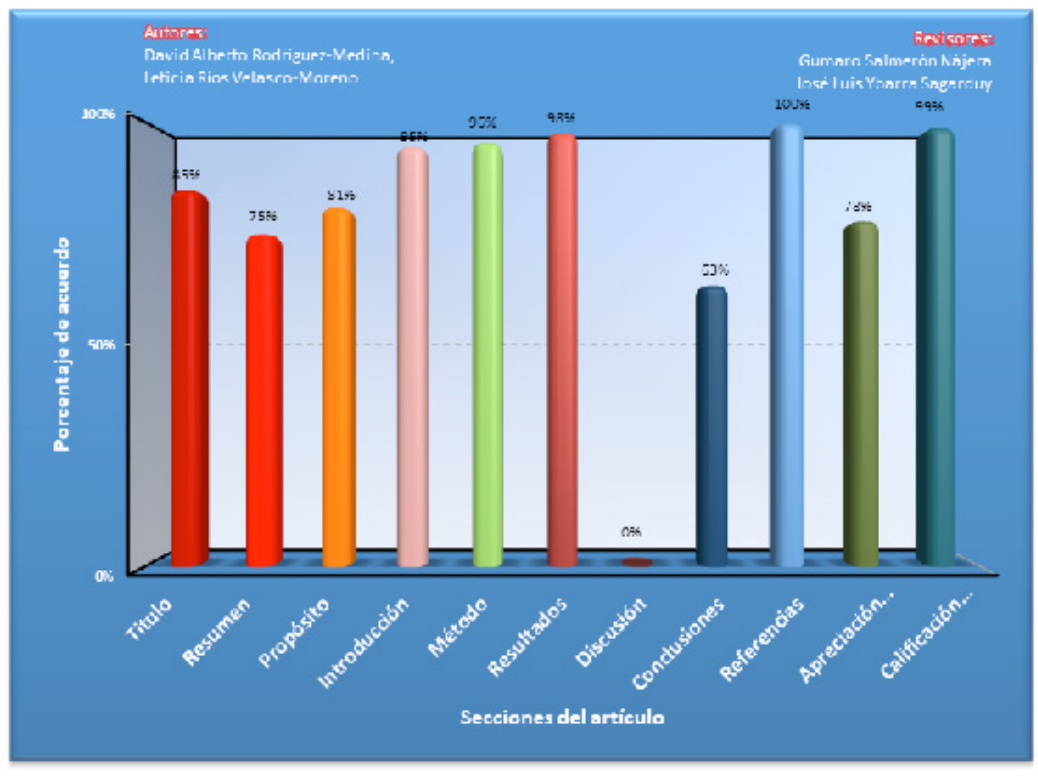




\section{Dimensión Cualitativa}

\begin{tabular}{|c|c|}
\hline Revisor 1 & Revisor 2 \\
\hline Gumaro Salmerón Nájera & José Luis Ybarra Sagarduy \\
\hline \multicolumn{2}{|c|}{ Título/Autoría } \\
\hline
\end{tabular}

Titulo/Autoría

Desde mi observación el título es adecuado.

Sería aconsejable aportar el correo del segundo autor

\section{Resumen}

Señalar los tiempos de evaluación previos y posteriores a la intervención.
El resumen sobrepasa las 150 palabras. En el resumen se menciona como una conclusión del estudio que se puede ampliar el manejo psicofisiológico con estrategias de aceptación y compromiso. Sin embargo, esto no se vuelve a retomar ni en la introducción ni en un apartado de conclusiones.

\section{Próposito del Estudio}

El estudio de caso no proporciona el lugar, el tiempo de intervención general y por sesión, tampoco proporciona las fechas de las pre y post evaluaciones y el tiempo transcurrido entre unas y otras, suceso fundamental para validar la eficiencia del tratamiento. La descrip- Nada que comentar ción del examen mental es escaso y se ignora el juicio hecho básico en un sujeto contrastorno bipolar tipo 1l, más aún si no está bajo tratamiento farmacológico como en el caso de estudio. 


\section{Revisor 1}

Revisor 2

Introducción

El estudio de caso no cuenta con datos que informen sobre las características bioquímicas y genéticas del trastorno bipolar tipo ll, y su incidencia en la depresión e hipomanía, dicho trastorno requiere por sus características intervención farmacológica, de lo contrario las evaluaciones estarán alteradas de acuerdo a 12/4/2019 Instrucciones de Dictaminación de un Manuscrito Enviado a la RDIPyCS cuved.unam.mx/campus/mod/ questionnaire/print.php?qid $=423 \&$ rid $=89203 \&$ courseid=316\&sec $=1$ 10/21 16 la etapa en que se encuentre el paciente, la remisión de los síntomas es sólo mediante la regulación de los químicos cerebrales, para posteriormente dar inicio a un proceso psicoeducativo. Existe la posibilidad de que los resultados obtenidos sean producto del estado depresivo o hipomaniáco del envaluado. Punto que debe revisarse por los investigadores.

En el artículo se denomina como intervención psicofisiológica a un paquete de entrenamiento integrado por relajación, estrategias cognitivo-conductuales y expresión emocional. Aunque en la introducción se mencionan brevemente los estudios de intervención cognitivo-conductuales en este tipo de pacientes, los 12/4/2019 Instrucciones de Dictaminación de un Manuscrito Enviado a la RDIPyCS cuved.unam.mx/campus/mod/questionnaire/ print.php?qid $=423 \&$ rid $=89198 \&$ courseid $=316 \&$ sec $=1$ $10 / 2116$ autores señalan que hay un desconocimiento sobre el efecto de las estrategias de relajación, el biofeedback y la expresión emocional en el trastorno bipolar. Sin embargo, no se hace una verdadera revisión de posibles estudios antecedentes sobre estos aspectos y de manera acelerada se menciona este vacío para justificar la intervención (p.e., hay revisiones que no se mencionan como "The Use of Narrative Therapy with Clients Diagnosed with Bipolar Disorder" Ngazimbi, Evadne E.; Lambie, Glenn W.; Shillingford, M. Ann, Journal of Creativity in Mental Health, v3 n2 p157-174 Jul 2008). Se sugiere una revisión más a fondo de las estrategias de intervención psicológicas usadas en pacientes con trastorno bipolar para justificar adecuadamente la aportación de este caso. Al ser un estudio de caso, no aplican los apartados de marco teórico, variables del estudio e hipótesis

\section{Método}

Anexar entrevista y observación clínica. Agregar fecha, lugar y tiempo de sesión y de la intervención. Agregar fecha, lugar y tiempo de la post evaluación, lo cual valida la intervención, debido a que es útil como parte de un proceso integral de intervención en el trastorno depresivo bipolar tipo 11. Agregar el apego a la ética y el consentimiento informado

Se recomienda dar un orden a la presentación del caso siguiendo las normas para redacción de casos clínicos recogidas en el artículo de Buela Casal, Gualberto; Sierra, Juan Carlos Normas para la redacción de casos clínicos International Journal of Clinical and Health Psychology, vol. 2, núm. 3, febrero-marzo, 2002, pp. 525-532. Falta información sobre las características psicométricas de los instrumentos de evaluación uasados y los tipos de análisis a realizar (cualitativo o estadístico), así como una descripción más detallada del mapa de patogènesis y del plan de intervención. 


\begin{tabular}{|c|c|}
\hline Revisor 1 & Revisor 2 \\
\hline \multicolumn{2}{|c|}{ Resultados } \\
\hline $\begin{array}{l}\text { Exponer la ficha técnica de los instrumentos, graficar que } \\
\text { puntos se mantienen, cuales varían y la fecha entre uno y otro. }\end{array}$ & Se deberían explicar mejor las tablas y figuras en el texto \\
\hline \multicolumn{2}{|c|}{ Discusión } \\
\hline $\begin{array}{l}\text { Anexar la parte bioquímica y genética del trastorno bi- } \\
\text { polar, la atención psiquiátrica y la trascendencia de la } \\
\text { parte psicofisiológica, en beneficio de la salud mental } \\
\text { en personas con trastornos bipolares. }\end{array}$ & No aplica el apartado de discusión para un estudio de caso \\
\hline \multicolumn{2}{|c|}{ Conclusiones } \\
\hline $\begin{array}{l}\text { Anotar el psicodiagnóstico realizado por los investiga- } \\
\text { dores. -Anotar el tiempo que transitó entre las primeras } \\
\text { y últimas evaluaciones. }\end{array}$ & $\begin{array}{l}\text { Se sugiere terminar el caso con un apartado de conclu- } \\
\text { siones donde se indiquen las limitaciones del estudio, las } \\
\text { contribuciones más importantes en relación a la investi- } \\
\text { gación antecedente, repercusiones prácticas o teóricas de } \\
\text { los resultados. }\end{array}$ \\
\hline \multicolumn{2}{|c|}{ Referencias } \\
\hline $\begin{array}{l}\text { Anexar las fichas técnicas y escalas de validez de los } \\
\text { instrumentos. }\end{array}$ & $\begin{array}{l}\text { Faltan más referencias sobre estudios antecedentes del uso } \\
\text { de estrategias de relajación, el biofeedback y la expresión } \\
\text { emocional en el trastorno bipolar. }\end{array}$ \\
\hline
\end{tabular}

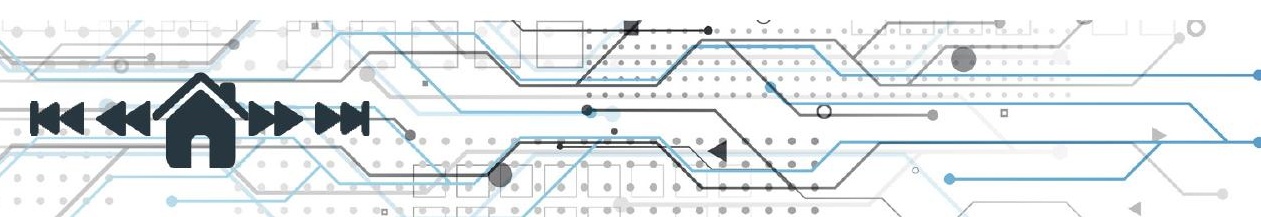


